\title{
T- Lymphocyte Subset (CD4 /CD8) Ratios of Breast Cancer Patients in Basra-Iraq and Baku-Azerbaijan
}

\author{
Noori Nasr Ghalib ${ }^{1 *}$, GM Nasrullayeva ${ }^{1}$, AY Qaziyev ${ }^{1}$, KH Al-Ali Jawad ${ }^{2}$
}

\begin{abstract}
Breast cancer is the most common cancer among the population as a whole and among females, yet it is highly curable if diagnosed at an early stage. Different methods are used to diagnose breast cancer. One of these methods features immunological tests using flow cytometry to determine T-lymphocyte (CD4/CD8) ratios in peripheral blood. One hundred patients with breast cancer ( 50 from Basra, Iraq, and 50 from Baku, Azerbaijan) confirmed to have breast cancer by histopathology were studied. Blood samples were collected from all patients before initiation of treatment and were used for analysis. The mean age of women from Basra was $51.2 \pm 10.7$ years and that of women from Baku was slightly higher at $54.8 \pm 12.2$. The mean CD4/CD8 ratio in Basra was 1.4 and in Baku was 1.8 with $\mathrm{P}$ value $<$ 0.05 . The percentage of Basra patients who have CD4/CD8 value less than 1 was $50 \%$, while the percentage for Baku patients was $24 \%(\mathrm{p}<0.05)$. While the CD4/CD8 T-lymphocyte ratio might be useful for early diagnosis in patients with breast cancer parallel with other confirmed tests factors involved in explaining variation between countries such as that observed here need to be taken into account.
\end{abstract}

Keywords: Breast cancer - CD4 /CD8 ratio - Basra - Baku - country variation

Asian Pac J Cancer Prev, 17, Cancer Control in Western Asia Special Issue, 175-177

\section{Introduction}

Lymphocytes are mobile cells, continuously recirculating between the blood and tissues, returning to the blood via the lymphatic system (Westermann 1990). The correlation between immune infiltrate and breast cancer has been widely investigated since the early work of Black (Black MM 1977)

A quantitative assessment of cell-mediated immunity is the measurement of subpopulations of T-helper and T- suppressor lymphocytes. Impaired cellular immunity can be associated with a depressed CD4/CD8 Ratio. The exact relation between the efficacy of T- cell-dependent immune mechanisms or between the extent and type of lymphocyte infiltration and tumor progression in carcinoma of the breast is a matter of debate (Liyange et al 2002 , Vagenopolous et al 2033). This interrelation is suspected of determining the influence of the immune system on the pathogenesis and progression of breast cancer (Macchetti et al, 2006)

The activation of CD8 cytotoxic T-cells has been regarded as a major antitumor mechanism of the immune system. Emerging evidence suggests that CD4 T-cells are required for the generation and maintenance of effective CD8 Cytotoxic and memory T-cells, a phenomenon known as CD4 $\mathrm{T}$ cell help.

CD4 T-cell help facilitates the optimal expansion, trafficking, and effector function of CD8 T cells, thereby enhancing tumor destruction (Yo-Ping Lai et al, 2011)

IN this study we decided to use CD Markers to evaluate the immune status of two different populations (Basra, Iraq and Baku, Azerbaijan).The present study is the first one that determine the immune response by immunophenotyping of lymphocytes between these countries that provide valuable diagnostic information for patients with breast cancer.

\section{Materials \& Methods}

A total of 100 peripheral blood specimens (50 from Basra \& 50 from Baku) with breast cancer were collected. Peripheral blood samples (TWO milliliters) collected from each patient by (EDTA) tubes for complete blood count, and CD Markers (by flow cytometry).

All cancer patients were subjected to histopathological investigations and before initiation of treatment were used for analysis. The Mean and P. value calculated for each parameter and Significant values are considered to occur at $\mathrm{p}<0.05$.

\section{Results}

The mean CD4 in BASRA was 0.9 and in Baku it was 0.8 with no significant difference (Figure 1). The Mean $\mathrm{CD} 8$ in Basra was 0.84 significantly higher $(\mathrm{P}<0.05)$ than among women in Baku (Figure 2).

${ }^{1}$ Azerbaijan Medical University, Baku, Azerbaijan, ${ }^{2}$ Basra Cancer Center, Member of Iraqi Cancer Board, Iraq.

*For correspondence: g.noori90@yahoo.com 


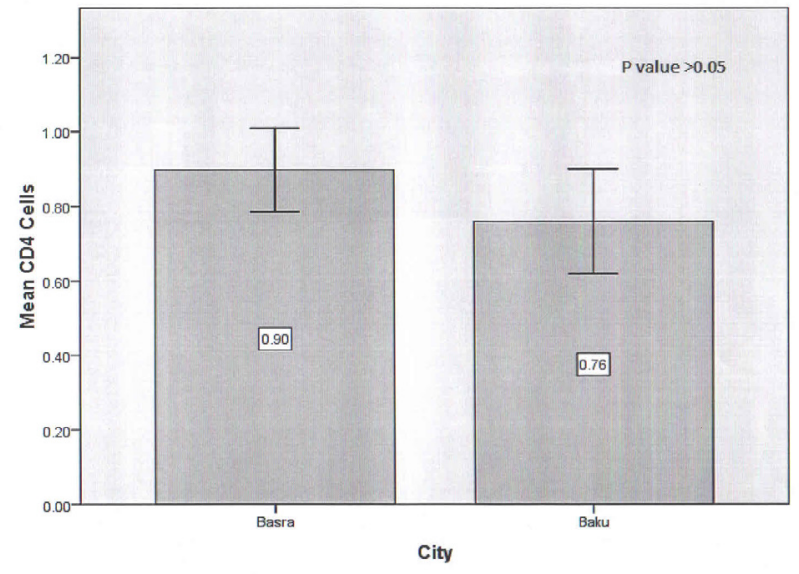

Error Bars: $95 \% \mathrm{Cl}$

Figure 1. The Mean CD4 in Basare and Baku

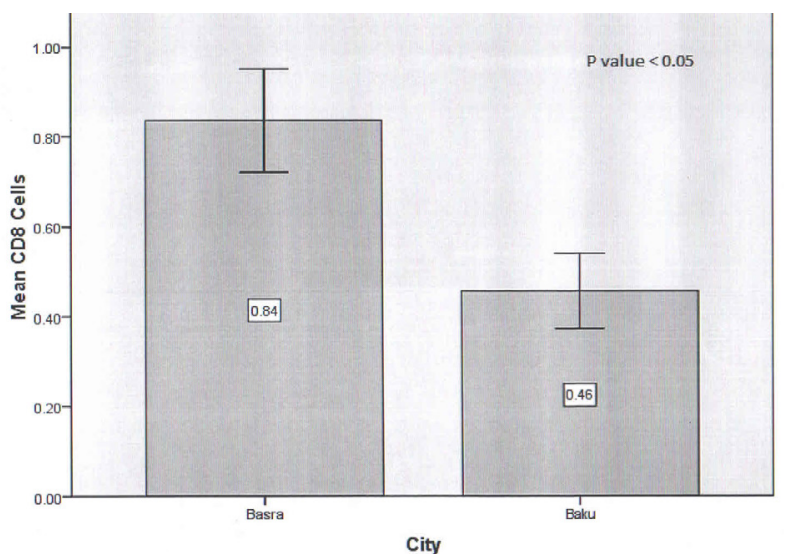

Figure 2. The Mean CD8 in Basare and Baku

Mean CD4/CD8 Ratio in Basra (1.38) and Baku (1.80) with $p$ value $<0.05$.(Figure 3 ). The percentage of Basra patients who have CD4/CD8 value lower than one is $50 \%$,while the percentage for Baku patients is $24 \%$ (p $(<0.05)$ (Figure 4)

\section{Discussion}

The study observed higher significant in level in CD8 value between Basra and Baku and the ratio of CD4/CD8 between two cities showed difference value and this was due to the differences in risk factors such as race ethnicity as well as genetic, and environmental status (Warne et al, 2014)

The ratio of CD4/CD8 $\mathrm{T}$ cells has been used as indicator for evaluating an individual immune function. In general the $\mathrm{CD} 4 / \mathrm{CD} 8$ ratio in healthy people is often more than one; nonetheless, in patients with terminal cancer, this ratio drops significantly.

Thus an inverted CD4/CD8 ratio in cancer patients is one of the $\mathrm{T}$ cell immune risk phenotypes that is associated with increased morbidity and mortality. Most studies have evaluated the composition of tumor infiltrate lymphocyte by immunohistochemistry (Mahmoud et al2011). Here we used flow cytometry, which is a more accurate technique (Farhad et al., 2015).

In conclusion, in early stages of breast cancer, the presence of $\mathrm{CD} 4$ and $\mathrm{CD} 8$ suggests that immune response

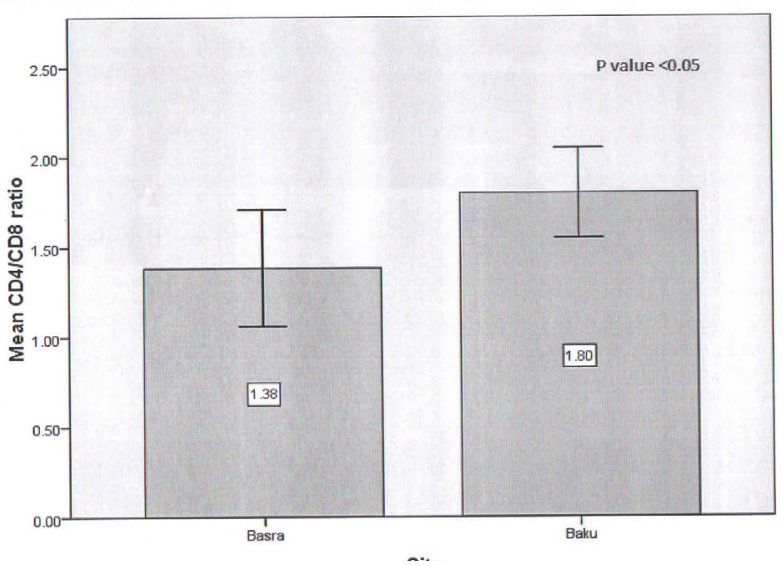

Figure 3. The Mean CD4/ CD8 Ratio in Basare and Baku

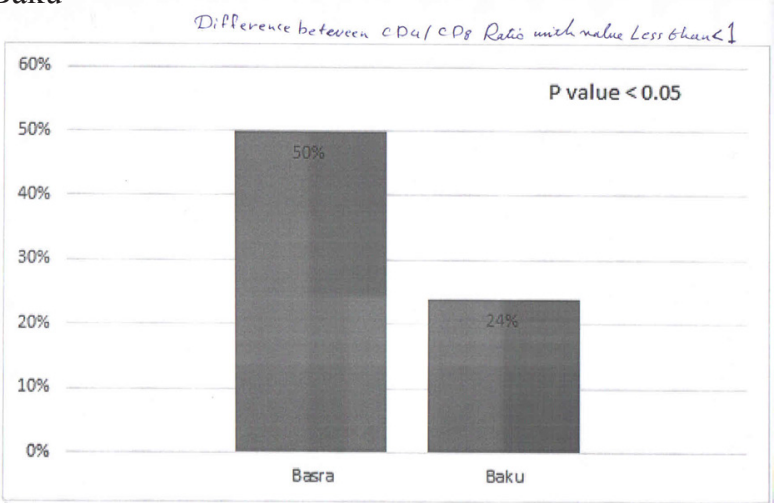

Figure 4. Difference between CD4/ CD8 Ratio in Basare and Baku

has an influence on the behavior of malignant breast tumors.

\section{References}

Black MM. (1977). Immunopathology of breast cancer. Pathobiol Annu, 7, 213-30

Farhad RR, Soheila A, Ramesh O, et al (2015). Comparative analysis of CD4,CD8 T cells in tumor tissue, lymph nodes and peripheral blood from patients with breast cancer. Iranian $B j$ 19, 35-44.

Liyange UK, Moree TT, Joo HG et al (2002). Prevalence of regulatory $\mathrm{T}$ cells is inceased in peripheral blood and tumor microenvironment of patients with pancreas or breast adenocarcinoma. J Immunol, 169, 2756-61.

Macchetti AH, Marana HRC, Silva JS, et al (2006). Tumor infiltrating CD4 T lymphocytes in early cancer reflect lymph node involvement. Cliinics, 61, 203-8.

Mahmoud SM, Paish EC, Powe DG, et al (2011). Tumor infiltrating CD8 lymphocytes predict clinical outcome in breast cancer. J Clinc Oncol, 29, 1949-55.

Vagenopoulo S, Lazaris AC, Markopolus C, et al (2003). Immunohistochemical evaluation of immune response in invasive ductal breast cancer of not-otherwise $=$ specified type. Breast, 12, 172-8.

Warne ET, Tamimi RM, Hughes ME, et al (2014). Racial and ethnic differences in breast cancer survival. J Clin Oncol, 33, 2254-61.

Westermann J, Pabst R (1990). Lymphocyte subsets in the blood: a diagnostic window on the lymphoid system. Immunol Today, 11, 406-10. 
Yo-Ping L, Chung-Jiuan J, Shu-ching C (2011). The roles of CD4

$\mathrm{T}$ cell in tumor immunity. $J$ Immunol, 6, 65-72. 